\title{
Evaluation of the Reliability of Hamular Notch-Incisive Papilla Plane (HIP) in Establishing Occlusal Plane
}

\author{
Dr Dipak Thapa \\ Department of Prosthodontics, Kantipur Dental College, Kathmandu, Nepal
}

Correspondence: Dr Dipak Thapa; email: drdthapa@hotmail.com

\section{ABSTRACT}

Introduction: There are various recommendations, using both soft tissue and hard tissue landmarks, for the determination of occlusal plane in dentistry. However, their reliability and accuracy has always been questioned. Hamular notch-Incisive papilla Plane (HIP) is one of such landmarks which is stated to be parallel to occlusal plane; however its reliability needs to be evaluated.

Objective: To find the relationship between hamular notch-incisive papilla plane and occlusal plane in dentulous subjects.

Materials \& Method: 48 dentate subjects with normal Class I occlusion participated in the study. Their maxillary impressions were made and casts were poured. Each cast was then mounted in the surveyor and HIP plane made parallel to the floor by tripoding method. With the cast in this relation, the vertical distance between HIP and various reference points of occlusal planes were measured using digital Vernier Caliper. Wilcoxon test was used to find the statistical difference $(p<0.05)$.

Result: During evaluation of data, none of the cast showed absolute parallelism between occlusal plane and HIP plane. The mean vertical distance between HIP and incisal edge (INC) was $6.44 \mathrm{~mm}$, whereas between HIP-6RMP and HIP-6LMP were $6.41 \mathrm{~mm}$ and $6.12 \mathrm{~mm}$ respectively. About $81 \%$ cases showed parallelism within the range of $2 \mathrm{~mm}$. 2-related sample statistic test showed no statistically difference $(p<0.05)$ between HIP-INC and HIP-6RMP; and HIP-INC and HIP-6LMP.

Conclusion: HIP plane tends to be parallel to occlusal plane and can be used as a clinical guideline in the determination of inclination of the occlusal plane.

Key words: hamular notch-incisive papilla plane, occlusal plane, surveyor

\section{INTRODUCTION}

According to The Glossary of Prosthodontic Terms, occlusal plane is the average plane established by the incisal and occlusal surfaces of the teeth.' Actually it is not the plane but represents the planar mean of the curvature of these surfaces.

Occlusal plane has great importance in dentistry. Changes in the plane of occlusion will modify the physical and functional relationship of the oral musculature leading to an alteration in function, comfort and the esthetic value. ${ }^{2-4}$

Numerous authors have proposed several landmarks to help define the level of occlusion plane. Camper plane and inter pupilary line are the usual morphologic guides used with the Fox plane in Complete Denture Prosthodontics. Other landmarks are the height of retro-molar pad, lateral borders of the tongue, position of the parotid papilla, commisures of the lips and buccinators grooves. ${ }^{5-8}$
Cooperman indicated that one could locate the anatomical landmarks of hamular notch and incisive papilla to orientate the hamular notch-incisive papilla (HIP) occlusal plane.? Sugaya et al suggested that, by using HIP plane one could quite predictably reproduce occlusal plane. ${ }^{10}$ So the purpose of this study was to identify the relation between these two planes using surveyor and thus assess the reliability of HIP in establishing the occlusal plane.

\section{MATERIALS AND METHOD}

A total of 48 dentate subjects were selected for this study. Most of them were dental students and some were patients who visited Department of Prosthodontics, Kantipur Dental College \& Hospital. Thorough clinical examination was done to ensure following inclusion criteria: complete permanent and healthy dentition, including the second molars with bilateral Angle Class I molar and canine relations; horizontal overlap and vertical overlap ranging from 2-4 $\mathrm{mm}$; no temporomandibular 
joint disorder; absence of extensive restorations or cuspal coverage; no previous or current orthodontic treatment; absence of anterior or posterior cross bite; and absence or minimal dental crowding.

Alginate impressions were made for each subjects and the casts were pour with Type III dental stone. The deepest point of the hamular notch and the center of the incisive papilla were marked with the color pencil. Each cast was mounted in the Marathon Surveyor and hamular notch-incisive papilla plane was made parallel to the floor by tripoding method; that is all three marked points were brought to same level and in contact with the fixed vertical arm.

At this point, the upper part of the surveying arm, where the spring is loaded, was measured using Vernier caliper and this was recorded under heading HIP (Figure 1). Then the surveying arm, not the horizontal arm, was moved up ward at the level of right central incisal edge (INC) and again the same area was measured. The same was done in relation to tip of mesiopalatal cusp of right first (6RMP) and left first maxillary molars (6LMP). These measurements were recorded under heading INC, 6RMP and 6LMP. Now by subtracting HIP from INC, 6RMP and $6 L M P$, the vertical distance between the HIP and incisal edge, HIP and mesio-palatal cusp of right first molar and HIP and mesio-palatal cusp of left first molar were calculated. If these measurements were equal at all three points, it meant that HIP and occlusal plane are absolutely parallel.

The data collected were statistically analyzed to test the equality of the mean differences using Wilcoxon nonparametric 2-related sample test, the significance level was set at $p<0.05$ for each analysis.

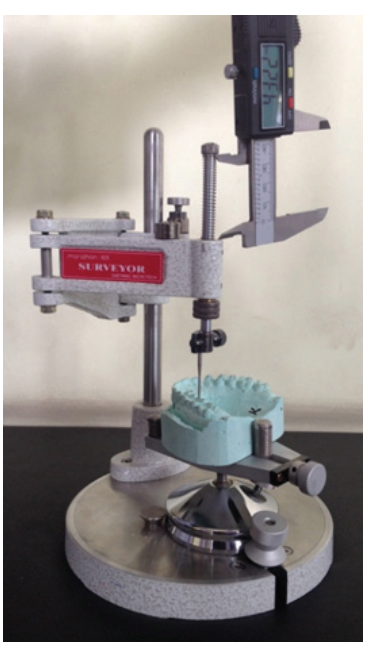

Figure 1: Surveyor with dental cast mounted and digital Vernier Caliper

\section{RESULT}

In the present study, none of the cases showed absolute parallelism between HIP plane and occlusal plane. However $81.25 \%$ of the cases showed parallelism within the range of $2 \mathrm{~mm}$. The mean vertical distance between HIP plane and incisal edge (INC) was $6.44 \mathrm{~mm}$, whereas between HIP and 6RMP, and HIP and 6LMP was $6.41 \mathrm{~mm}$ and $6.12 \mathrm{~mm}$ respectively (Table 1). Keeping mean vertical distance HIPINC as standard, the mean difference between HIP-6RMP and HIP-6LMP were $0.03 \mathrm{~mm}$ and $0.32 \mathrm{~mm}$.

Statistical analysis showed no statistically significant difference ( $p>0.05$ ) between HIP-INC and HIP-6RMP (Pair1), and HIPINC and HIP-6LMP (Pair 2). But when the same test was done between HIP-6RMP and HIP-6LMP (Pair 3), statistically significant difference $(p<0.05)$ result was obtained (Table 2$)$.

Table 1: Distance between HIP and Occlusal plane in incisal, right first moral and left first molar region.

\begin{tabular}{|l|c|c|c|}
\hline \multicolumn{1}{|c|}{ Groups } & Mean & SD & Min \\
\hline HIP- INC & 6.44 & 1.54 & 4.01 \\
\hline HIP-6RMP & 6.41 & 0.98 & 4.85 \\
\hline HIP-6LMP & 6.12 & 0.76 & 8.90 \\
\hline
\end{tabular}

Table 2: Wilcoxon test statistics for difference between the pairs

\begin{tabular}{|c|c|c|c|c|c|}
\hline & $\mathbf{N}$ & Mean & SD & SEM & $p$-value \\
\hline \multicolumn{6}{|l|}{ Pair 1} \\
\hline HIP-INC & 48 & 6.445 & 1.544 & 0.222 & \multirow{2}{*}{0.712} \\
\hline HIP-6RMP & 48 & 6.413 & 0.983 & 0.141 & \\
\hline \multicolumn{6}{|l|}{ Pair 2} \\
\hline HIP-INC & 48 & 6.445 & 1.544 & 0.222 & \multirow{2}{*}{0.214} \\
\hline HIP-6LMP & 48 & 6.125 & 0.764 & 0.110 & \\
\hline \multicolumn{6}{|l|}{ Pair 3} \\
\hline HIP-6RMP & 48 & 6.413 & 0.983 & 0.141 & \multirow{2}{*}{$0.034^{*}$} \\
\hline HIP-6LMP & 48 & 6.125 & 0.764 & 0.110 & \\
\hline
\end{tabular}

*statistically significant at $p<0.05$ 


\section{DISCUSSION}

It is evident from the literature that there are controversies regarding the location and orientation of the occlusal plane. ${ }^{4}$ Reliable landmarks that can better guide the clinician in establishing occlusal plane are very necessary. HIP was reported in the literature to be used in establishing the occlusal plane. ${ }^{11,12}$ As these landmarks appeared to be least affected by residual ridge resorption and are easily visible, it could be of great help if they could be used to orient the occlusal plane.

Rich, Karkaziz and Polyzois ${ }^{11,12}$ observed parallel relation between HIP and the occlusal plane; however they insisted on further evaluation considering the discrepancies in their methodology. Karkazis and Polyzois applied cephalometry to investigate the relationship between occlusal plane and HIP plane.

Fu et $a 1^{4}$ found $2.61^{\circ} \pm 0.81$ angle between the occlusal plane and HIP in Taiwanese subjects. Similar result of $1.60^{\circ} \pm 2.2$ was noted by Sugaya et $\mathrm{a}^{10}$ in Japanese. Both studies used threedimensional precise measuring devices to measure the angle between occlusal plane and HIP, and concluded that HIP tends to be more parallel to occlusal plane and can be used as a clinical guide.
In this study, dental surveyor was used and the HIP was kept parallel to the floor by tripoding method. Then the vertical height was measured at three points: INC, 6RMP and 6LMP. The statistical result obtained was little different from other researchers. The posterior part of the occlusal plane was parallel with the HIP but the anterior part was not. At the same time more than $80 \%$ subjects had HIP parallel to occlusal plane within the range of $2 \mathrm{~mm}$. This suggests that HIP tends to be parallel to the occlusal plane; however further investigations with larger sample are required to establish this concept.

\section{CONCLUSION}

Hamular notch-incisive papilla plane (HIP) tends to be parallel to occlusal plane and can be used as a clinical guideline in the determination of inclination of occlusal plane.

\section{ACKNOWLEDGEMENT}

The author heartily acknowledge Dr Sujita Shrestha for helping in statictical analysis and Dr Kushal Das Maharjan, Intern for helping in data collection.

\section{OJN}

\section{REFERENCES}

1. The Glossary of Prosthodontics Terms. J Prosthet Dent 1999; 81:39-110.

2. Ferrario VS, Sforza C, Serrao G, et al: Three-dimensional assessment of the reliability of a postural face bow transfer. J Prosthet Dent 2002; 87:210-215

3. Jayachandran S, Ramachandran R, Varghese R: Occlusal Plane Orientation: A Statistical and Clinical Analysis in Different Clinical Situations. Journal of Prosthodontics 2008; 17:572-575.

4. Fu PS, Hung C, Hong J, et al: Three-dimensional analysis of the occlusal plane related to the hamular incisive papilla occlusal plane in young adults. Journal of Oral Rehabilitation 2007; 34:136-140.

5. Acharya B: Comparison of Maxillary occlusal plane with various craniofacial reference lines in Nepalese and Indian young adults. Orthod J Nepal 2011; 1:16-19.

6. Yasaki M: The height of the occlusion rim and the interocclusal distance. J Prosthet Dent 1961; 11:26-31.

7. Ismail YH, Bowman JF: Position of the occlusal plane in natural and artificial teeth. J Prosthet Dent 1968; 20:407-411.

8. Foley PF, Latta GH: A study of the position of the parotid papilla relative to the occlusal plane. J Prosthet Dent1985; 53:124-126.

9. ooperman HN. HIP plane of occlusion in oral diagnosis. Dent Surv. 1975; 51:60-62.

10. Sugaya K, Suminokuro S, Ohira H, et al. A study on correlation ship among various planes in dental prosthetics.J Jpn Prosthodontic Soc. 1986; 30:1347-1358.

11. Rich H:Evaluation and Registration of HIP plane of Occlusion.Aust Dent J 1982; 27:162-168.

12. Karkaziz HC, Polyzois GL: Cephalometrically predicted occlusalplane: implication in removable prosthodontics. J Prosthet Dent 199: 65:258-264. 